\title{
Primary amoebic meningoencephalitis
}

\author{
A R R CAIN, P F WILEY, BETTY BROWNELL, AND D C WARHURST \\ Department of Paediatrics, Royal United Hospital, Bath, Department of Neuropathology, Frenchay \\ Hospital, Bristol, and Amoebiasis Unit, Hospital for Tropical Diseases, London
}

SUMMARY The third case in the UK of primary amoebic meningoencephalitis is reported; it affected an 11-year-old girl. Six days before admission the girl had swum in a pool fed by hot spring water in which the causative agent Naegleria fowleri was found. Early treatment with amphotericin B would seem to offer the only hope of recovery in this almost uniformly fatal infection.

Primary amoebic meningoencephalitis (PAM) was first reported by Fowler and Carter ${ }^{1}$ in south Australia, 3 of their 4 patients being children. Other cases have been documented in America, Belgium, Czechoslovakia, and New Zealand. Symmers ${ }^{2}$ recorded 2 retrospective cases from the UK. The first case of PAM in the UK to be diagnosed in life was reported in $1970 .^{3}$ A young boy died from pyogenic meningitis after splashing about in a warm puddle. Amoebae (Naegleria sp.) were found in the cerebrospinal fluid (CSF). His brother recovered after a non-meningitic pyrexial illness. Naegleria sp. was not seen in the CSF but grew in culture. We describe a further case of PAM which occurred in Bath in July 1978.

\section{Case report}

An 11-year-old girl was admitted with a 3-day history of headache, and a 1-day history of pyrexia, vomiting, and blurred vision. Six days previously she had swum in an indoor pool fed by natural warm spring water. She was drowsy with slight neck stiffness and a temperature of $38 \cdot 5^{\circ} \mathrm{C}$. Eye movements and fundi were normal.

Investigations. Haemoglobin $13.4 \mathrm{~g} / \mathrm{dl}$, white blood cells $20.4 \times 10^{9} / 1$ with neutrophils $90 \%$. A lumbar puncture showed turbid CSF under pressure of more than $220 \mathrm{~mm}$. The CSF protein was $5.5 \mathrm{~g} / \mathrm{l}$ $(550 \mathrm{mg} / 100 \mathrm{ml})$ and glucose $<0.5 \mathrm{mmol} / 1(9 \mathrm{mg}$ / $100 \mathrm{ml}$ ), (blood glucose $12.4 \mathrm{mmol} / 1 ; 223 \mathrm{mg} / 100$ $\mathrm{ml}$ ). No organisms were seen. There were 500 red cells $\times 10^{6} / 1$ and 4000 white cells $\times 10^{6} / 1,90 \%$ of the cells being reported as polymorphs.

\section{Progress}

A diagnosis of pyogenic meningitis was made and treatment with intravenous penicillin, sulphadimidine, and chloramphenicol was started. Her condition deteriorated during the next few hours and intramuscular phenytoin was given. Thirteen hours after admission she had a short convulsion and 2 hours later she had a more-prolonged one. She was given intravenous diazepam and intramuscular paraldehyde. Her fundi remained normal but she was given mannitol intravenously to reduce presumed cerebral oedema. Twenty hours after admission respiratory arrest occurred necessitating mechanical ventilation. Dexamethasone was started. By then she was unresponsive with very sluggish, midpoint pupils.

Thirty-six hours after admission the CSF and blood cultures showed no growth and a repeat lumbar puncture was done. The CSF was turbid and very slow to drain. Direct microscopical examination of the CSF in a cell counting chamber (Mr Alan Yates) showed numerous motile amoebae. Discussions with staff at the Communicable Disease Surveillance Centre and the Amoebiasis Unit led to a change in treatment. Chloramphenicol, penicillin, and dexamethasone were stopped and amphotericin B $0.5 \mathrm{mg} / \mathrm{kg}$ a day by single 6-hourly infusion started. Sulphadimidine was continued with the addition of rectal metronidazole. A ventricular catheter was inserted through a frontal burr hole (Mr A V Hulme) for the administration of $0.15 \mathrm{mg}$ amphotericin $\mathrm{B}$. The ventricular CSF contained numerous motile amoebae. During the next 2 days she remained comatose with fixed pupils. It had been planned to increase the dosage of amphotericin B to $1 \mathrm{mg} / \mathrm{kg}$ a 
day but in view of a poor urinary output and rising blood urea $0.6 \mathrm{mg} / \mathrm{kg}$ a day was the maximum achieved.

On day 3 a further lumbar puncture was done and $0.05 \mathrm{mg}$ amphotericin B given. The CSF showed fewer amoebae, all non-motile, there being 200 red cells and 115 white cells $\times 10^{6} / 1$. Serum taken at this time was subsequently examined for immunoglobulins which were found to be present in normal amounts. The levels were IgA $1.5 \mathrm{~g} / \mathrm{l}, \operatorname{IgG} 10.8 \mathrm{~g} / \mathrm{l}$, and $\operatorname{IgM} 1.9 \mathrm{~g} / \mathrm{l}$. The same serum showed no reaction at a dilution of one-eighth in a fluorescent antibody test against Naegleria fowleri.

On day $4 \mathrm{CSF}$ obtained from the ventricular catheter before the instillation of $0.1 \mathrm{mg}$ amphotericin B showed no motile amoebae and a culture was negative. However the patient remained deeply comatose and she died on day 5 after cardiac arrest.

\section{Necropsy}

Permission for a full necropsy was not obtained but consent was given for the brain to be removed. The brain had been sliced before fixation and the slices showed generalised swelling and softening. A relatively mild leptomeningeal exudate was present throughout, particularly over the cerebellum and brainstem. No focal lesions were present in the cerebrum or hindbrain.

Representative sections from the cerebrum, cerebellum, and brainstem showed an inflammatory meningeal exudate, the cells being almost entirely mononuclear or lymphocytic. Numerous amoebae, staining poorly with routine methods, were present among the inflammatory cells. Infiltration of the nervous tissue was also present in the cerebral hemispheres and cerebellum, with great masses of parasites invading the tissue along the VirchowRobin spaces and spreading in the grey and white matter. Relatively little in the way of an inflammatory cell infiltrate accompanied these invading organisms (Fig. 1). In addition to the widespread meningoencephalitis, all parts of the nervous system showed histological changes attributable to cerebral anoxia.*

\section{Identification}

CSF from the patient was examined (Dr P G Mann, Public Health Laboratory Bath, and Dr Suzanne Clarke, Public Health Laboratory Bristol) and the organism was provisionally identified as Naegleria

*Frenchay Hospital, Neuropathology Department No NP7390.

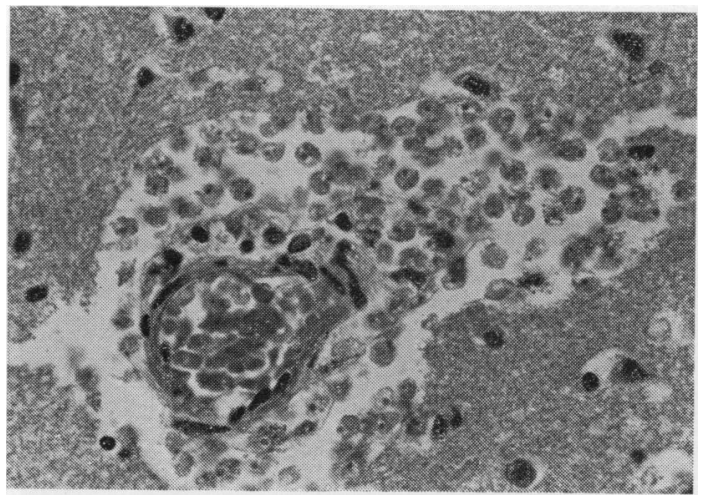

Fig. 1 A small vessel in the cortex of the frontal lobe surrounded by amoebae. Note the almost complete absence of inflammatory cells. Haematoxylin and eosin $\times 300$.

sp. Samples were posted, together with cultures, to the Amoebiasis Unit. Three isolates were made from the CSF, and amoebae grown from these samples were identical. Direct observation showed them to be 15 to $20 \mu \mathrm{m}$ in diameter when rounded, and in locomotion having an elongated sluglike form and a single lobose pseudopodium. Growth took place on agar spread with Klebsiella aerogenes, rapidly at 43 and $41^{\circ} \mathrm{C}$, more slowly at 37 and $35^{\circ} \mathrm{C}$, and very slowly at $25^{\circ} \mathrm{C}$. Dr Clarke grew the amoeba in RK-13 cell cultures, where it caused a cytopathic effect. When agar-grown trophozoites were exposed to distilled water, they changed into the biflagellate form within 2 hours at $37^{\circ} \mathrm{C}$. The cysts, produced readily on agar, were spherical, 9 to $10 \mu \mathrm{m}$ in diameter, without detectable pores. Preparations of trophozoites, fixed in acetone, reacted in the indirect fluorescent antibody test at titres of $1 / 512$ to $1 / 1024$ with specific anti- $N$. fowleri serum (prepared in rabbits).

Suspensions of amoebae were instilled intranasally into 10 mice. Controls were also set up using $K$. aerogenes suspension. All the mice inoculated were dead within 10 days; the controls survived. Cultures made from the brains of dead mice grew typical $N$. fowleri amoebae, and sections confirmed that the amoebae had invaded the brain. It was concluded that the organism was identical in all respects with a pathogenic strain of $N$. fowleri.

To confirm the presence of $N$. fowleri in the patient's brain paraffin sections were dewaxed, rehydrated, and examined by the indirect fluorescent antibody test, using anti- $N$. fowleri serum. Very large numbers of fluorescing amoebae were detected in material from the inferior surface of the left 


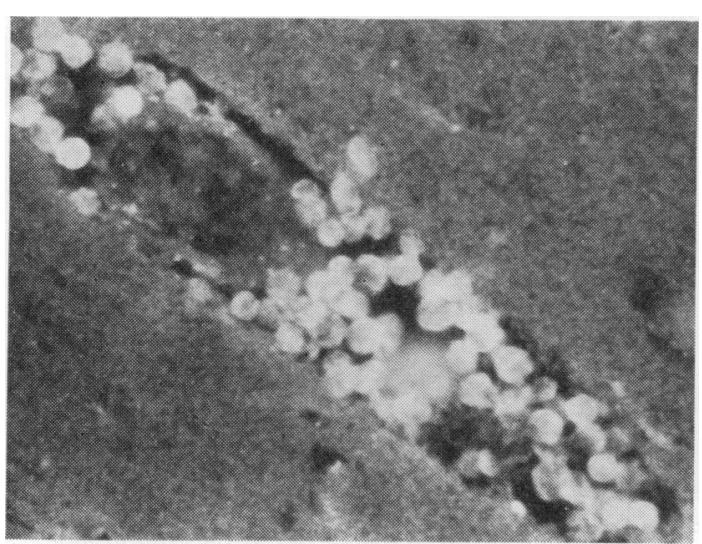

Fig. 2 Section from inferior surface of frontal lobe stained by the indirect fluorescent antibody technique using antiserum against $N$. fowleri (HBI strain). This shows the accumulation of fluorescing amoebae in the enlarged Virchow-Robin space.

frontal lobe (Fig. 2), where invasion and degeneration were so advanced that even the uninvaded tissue, in parts, seemed to be permeated by amoebic antigen, giving a high background fluorescence. In addition, many amoebae were noted in the cerebellar hemispheres. There was a cellular response in these areas, but occurring well behind the path of amoebic invasion. This appeared to have taken place via the subarachnoid space and into the Virchow-Robin spaces. Amoebae were found in the Virchow-Robin spaces and also in the brain parenchyma, well ahead of any cellular responses, in the superior frontal lobe $(L)$, parietal lobe (R), temporal lobe (L), hippocampus (L), basal ganglia (R), and medulla.

$N$. fowleri have been isolated from the thermal spring water that supplied the pool in which the girl had been swimming before her fatal illness. Details of this investigation will be the subject of a separate publication.

\section{Discussion}

There have now been over 80 case reports of primary amoebic meningoencephalitis from around the world and the outcome has been almost uniformly rapidly fatal. Many of these patients have become infected in swimming pools supplied with naturally heated spring water and it is of interest that the hot spring supply to this particular swimming pool has a temperature of $48^{\circ} \mathrm{C}$ at source.

This is the second recent case report in Britain of PAM caused by Naegleria sp., and it is possible that there have been other cases diagnosed as 'pyogenic meningitis, no organism isolated'. The course and outcome were unfortunately typical. Amphotericin B given after early diagnosis may offer some hope, and recently a successfully treated case has been reported from the USA where a combination of amphotericin, miconazole, and rifampin was used. ${ }^{4}$ Amphotericin $B$ is amoebicidal in vitro and in vivo, ${ }^{5}$ and in our case the amoebae in the CSF lost their motility after treatment. The drug may have influenced the recovery of the second case of Apley et $a .^{3}$

$N$. fowleri is a thermophilic organism, and its distribution is limited by the sensitivity of the cysts to desiccation and the need for a warm environment for growth. The amoebae grow only slowly at $25^{\circ} \mathrm{C}$, but at temperatures between 37 and $45^{\circ} \mathrm{C}$ growth is abundant. The trophozoites are reported to survive for 30 minutes at $51^{\circ} \mathrm{C} .^{6}$ Infection probably depends on the number of trophozoites and flagellates (the infective stages) present in water inhaled into the nose which is determined by the type of swimming and the concentration of or janisms in the water. The route of invasion is almost certainly through the olfactory neuro-epithelium. ${ }^{5}$ Most cases have occurred in children and young adults and it is possible that individual host susceptibility may be an important factor. Cursons et al. ${ }^{7}$ reported a case of PAM with a low serum IgA but the level in the patient reported here was normal.

The neuropathological findings in this case of PAM are similar to those described, for example, by Carter, ${ }^{5}$ though they are vastly different from those in the previous Bristol case, ${ }^{3}$ where there was a considerable cellular exudate, but only very few parasites. The lack of cellular response in the brain, in our case, was probably, at least in part, due to the anoxic necrosis which had occurred some time before the cardiac arrest.

Symmers $^{2}$ deduced that PAM occurred in the British Isles by demonstrating that a 1909 pathological specimen, previously labelled as 'meningitis carcinomatosa', was a case of amoebic meningoencephalitis. The brain was described as that of a "lad of Essex' but no clinical details were known and it is not certain that he became infected in Britain. Symmers also described a 10-year-old girl who died from PAM in 1937 in Belfast; she had been rescued from drowning at a public swimming place in the Belfast city waterworks 10 days previously. The temperature of the water was not recorded.

We should like to emphasis that PAM occurs in the UK and that prompt diagnosis and treatment with amphotericin B at present offers the only chance of recovery. The disease should be borne in mind in presumed pyogenic meningitis which does not 
respond to normal antibiotics and in which a high CSF white count, with no organisms, is found.

The appearance of $N$. fowleri in Gram- and Wayson-stained films of CSF has recently been described $^{8}$ and it was recommended that if similar structures were seen in the stained film, the examination of wet films was indicated. A high index of suspicion is particularly important if there is a history of swimming in, or just contact with, water which may be contaminated.

We thank Mr A Carman for carrying out the mouse pathogenicity tests, and Mrs A Birch for secretarial help.

\section{References}

1 Fowler M, Carter R F. Acute pyogenic meningitis probably due to Acanthamoeba sp. A preliminary report. Br Med J 1965; ii: 740-2.

2 Symmers W St C. Primary amoebic meningoencephalitis in Britain. Br Med $J$ 1969; iv: 449-54.
${ }^{3}$ Apley J, Clarke S K R, Roome A P C H, et al. Primary amoebic meningoencephalitis in Britain. Br Med J 1970; i: $596-9$.

4 Primary amoebic meningoencephalitis. California, Florida, New York. Morbid Mortal Weekly Report 1978; 27: No 37, 343-4.

5 Carter R F. Primary amoebic meningoencephalitis: an appraisal of present knowledge. Trans $R$ Soc Trop Med Hyg 1972; 66: 193-213.

6 Chang S L. Resistance of pathogenic Naegleria to some common physical and chemical agents. Appl Environ Microbiol 1978; 35: 368-75.

7 Cursons R T M, Keys E A, Brown T J, Learmonth J, Campbell C, Metcalf $P$. Letter:IgA and primary amoebic meningoencephalitis. Lancet 1979 ; i: $223-4$.

8 Boyle A L, Friedman T A, Braunstein H, Tomasulo M. Letter: Rapid diagnosis of primary amoebic meningoencephalitis due to Naegleria. Detection of organisms with bacterial stains. J Clin Pathol 1979; 32: 306-7.

Correspondence to Dr A R R Cain, Department of Paediatrics, Royal United Hospital, Combe Park, Bath BA1 3NG.

Received 9 October 1979 